\title{
ESSENTIAL LEADERSHIP DOMAINS AND STRATEGIES TO ENHANCE LEADERSHIP SKILLS AMONG UNDERGRADUATE MEDICAL STUDENTS: A MIXED METHODS STUDY
}

\author{
Syed Muslim Abbas, Abid Ashar* \\ University of Birmingham, Birmingham, United Kingdom, *Fatima Memorial Hospital/College of Medicine \& Dentistry, Lahore Pakistan
}

\begin{abstract}
Objective: To explore medical undergraduate students' perceptions pertaining to the key domains of leadership and highlight strategies to enhance leadership skills in undergraduate studies.

Study Design: Mixed methods study.

Place and Duration of Study: Fatima Memorial Hospital College of Medicine \& Dentistry, Lahore, from Sep to Oct 2019.

Methodology: A total of 207 medical students were recruited from the first and final year by purposive sampling to fill out a structured questionnaire. Three focus group discussions were conducted comprising of twelve students in each group. All focus groups were audio-recorded, anonymized and transcribed verbatim before the analysis by framework analysis technique.

Results: Total fifty percent of the first-year students were of the view that ensuring patient safety was an important component of the leadership domain of improving services whereas only $11 \%$ responded as critically evaluating as a component of this domain. The themes that emerged pertaining to the strategies to enhance leadership skills included increasing awareness, role models depicting change, cultural challenges, and supplementing existing practices.

Conclusions: The validated essential domains of leadership and its associated competencies should be streamlined strategically and prioritized in accordance with the level of training of the undergraduate medical students. A multicentre study is suggested to gather data at the national level which could inform further development of inclusion of leadership domains within undergraduate MBBS curriculum.
\end{abstract}

Keywords: Competencies, Leadership domains, Leadership skills, Medical students.

How to Cite This Article: Abbas SM, Ashar A. Essential Leadership Domains and Strategies to Enhance Leadership Skills Among Undergraduate Medical Students: A Mixed Methods Study. Pak Armed Forces Med J 2021; 71(5): 1791-1796. doi: https://doi.org/10.51253/pafmj.v71i5.3760

This is an Open Access article distributed under the terms of the Creative Commons Attribution License (https://creativecommons.org/licenses/by-nc/4.0/), which permits unrestricted use, distribution, and reproduction in any medium, provided the original work is properly cited.

\section{INTRODUCTION}

Leadership is a complex concept, which may contextualize in accordance with the operational settings and predetermined objectives at hand ${ }^{2,3}$. Healthcare leadership has been recognized in recent times as an essential component for improving the quality of care, optimal healthcare performance, efficient teamwork and strengthening health systems ${ }^{46}$. To incorporate leadership competencies strategies have been devised globally, particularly in the USA to integrate the essential skills-based training in undergraduate medical curriculum and ongoing training of Physicians 3,7 . Moreover, systematic review ${ }^{8}$, of research studies based on introducing leadership competency module among medical professionals suggest that such interventions yield positive results in changing attitudes, beliefs and competency levels. With changing trends in healthcare and the burden on weakened health infrastructures and resource limitations leadership competencies are targeted at all levels of training ${ }^{9,10}$. Leadership

Correspondence: Dr Syed Muslim Abbas, University of Birmingham, Birmingham, United Kingdom

Received: 22 Jan 2020; revision received: 04 Mar 2020; accepted: 04 Mar 2020 philosophies are complex demanding a diversified and collaborative approach to match the level of training of the health care professionals to which they are aimed $a^{2}$. Therefore, it is insightful to study the current status and development of leadership competencies among undergraduate medical students.

Leadership domains have been internationally defined and their associated competencies have been emphasized to be included in the undergraduate curriculum and capacity building or training of healthcare professionals ${ }^{3,7-10}$. To ensure successful implementation it is necessary to devise strategies that are tailored according to the training needs and should build on the existing competencies ${ }^{8}$. Such strategies can be country-specific considering the socio-cultural aspect which has been identified as an important indicator in determining favourable outcomes in various leadership domains 2,10 .

Therefore, with the various existing strategies and the validation of various competencies associated with Leadership, it is worthwhile to explore the perceptions among the medical undergraduates. Moreover, identification and prioritization of key indicators can be 
useful for successful implementation of leadership skills in undergraduate medical curriculum particularly from a developing health country perspective. Pakistan has a weak health infrastructure and requires a healthcare workforce with essential leadership skills. The study aims to explore medical undergraduate students perceptions pertaining to the key domains of leadership and its associated competencies and highlight strategies to enhance leadership skills to promote practice in the clinical and teaching environment as they are the instigators of change and the future of healthcare in Pakistan.

\section{METHODOLOGY}

The study had a mixed methods study design. Quantitative and qualitative data from medical undergraduate students was collected from September to October 2019. The perspectives and the input within the same institute ensured homogeneity of the data collected and analysis. The study was conducted in Fatima Memorial Hospital College of Medicine \& Dentistry which is a medical institute in Lahore with a tertiary care teaching hospital attached with the campus. The sampling strategy was non probability convenience sampling.

Inclusion Criteria: All the students from first and final year present on the day of the study were recruited.

Exclusion Criteria: Non-consenting individuals were excluded.

Focus group discussion of students were conducted among groups of 12 students each from first and final year MBBS. For the quantitative data collection the standardized validated questionnaire ${ }^{11-19}$, encompassing the five major domains of leadership and its associated competencies was utilized. The data collection procedure was confined within the institute. Views and opinions other than the proposed sample were not included in the analysis or discussion component of the study.

The questionnaire designed for collection for the quantitative data based on the five major domains and their subsequent categories was based on the leadership competencies defined by National Health System United Kingdom holistically encompassing medical students, practitioners and senior faculty ${ }^{18-21}$. The validated questionnaire was selected because it was formulated by consensus with many clinicians across a wide cross section of settings. The framework has been pilot tested and critically appraised since its inception in 2011 making it a valid and reliable tool to assess leadership competencies. The questionnaire encompasses five major domains of leadership competencies and its associated competencies. The five domains include; Demonstrating Personal qualities, working with others, managing services, improving services and setting direction ${ }^{21}$. The data for quantitative data was collected by distributing the validated questionnaire among the students of first and final year MBBS students.

The Focus group discussions were conducted utilising a Focus group guide with predefined questions keeping in view the key principles of framework analysis'22. Three focus group discussions were conducted comprising of 12 students of first and final year medical students in each group. The focus group discussions were conducted in closed settings and were recorded digitally. The focus groups lasted between 46-60 minutes. All focus groups were audio recorded, anonymized and transcribed verbatim before the analysis. Additional field notes were also taken by lead researcher MA. For reporting of the qualitative component, the study is guided by 'The Consolidated Criteria for Reporting Qualitative Studies (COREQ) 32 item checklist ${ }^{23}$, to ensure transparency.

Quantitative data was analysed using SPSS-21. Frequency distributions are presented in tables across the cohorts of first and final year medical students. The qualitative data collected through focus group discussion was analysed using framework analysis ${ }^{21}$, to generate important themes within the board structural framework of the domains of leadership competencies. This method of qualitative data analysis can be argued to be the most appropriate analytical approach and has recently become a very popular approach in management and analysis of data in health related research ${ }^{22}$.

\section{RESULTS}

The socio-demographic details of the students who participated in the study are provided in Table. There were $42(39 \%)$ male students and 65 (61\%) female students from 1st-year MBBS that participated in the survey. Whereas, $43(43 \%)$ male students and 57 (57\%) females participated in the survey from final year MBBS (Table). A significant percentage of students $(79 \%)$ were of 19 years of age in 1st year and an equal number of students had either completed local inter-mediate exams or GCE A levels prior to starting the undergraduate MBBS program. The socio-demographic details of the cohort of first and final year students are provided in detail in Table. 
Table: Socio-demographic details of the participants.

\begin{tabular}{|c|c|c|}
\hline 1 Year MBBS Students & Frequency (n) & Percentage $(\%)$ \\
\hline \multicolumn{3}{|l|}{ Age } \\
\hline 18 years & 8 & 7 \\
\hline 19 years & 79 & 74 \\
\hline 20 years & 18 & 17 \\
\hline$>20$ years & 2 & 2 \\
\hline Total & 107 & 100 \\
\hline \multicolumn{3}{|l|}{ Gender } \\
\hline Male & 42 & 39 \\
\hline Female & 65 & 61 \\
\hline \multicolumn{3}{|l|}{ Previous Education } \\
\hline Matric/FSc Pre Medical & 45 & 42 \\
\hline O/A Levels & 47 & 44 \\
\hline SAT (High School) & 13 & 12 \\
\hline Other & 2 & 2 \\
\hline Total & 107 & 100 \\
\hline Final Year MBBS Students & Frequency (n) & Percentage (\%) \\
\hline \multicolumn{3}{|l|}{ Age } \\
\hline 23 years & 2 & 2 \\
\hline 24 years & 81 & 81 \\
\hline 25 years & 11 & 11 \\
\hline$>25$ years & 6 & 6 \\
\hline \multicolumn{3}{|l|}{ Gender } \\
\hline Male & 43 & 43 \\
\hline Female & 57 & 57 \\
\hline \multicolumn{3}{|l|}{ Previous Education } \\
\hline Matric/ FSc pre Medical & 60 & 60 \\
\hline O/A Levels & 28 & 28 \\
\hline SAT (High School) & 10 & 10 \\
\hline Other & 2 & 2 \\
\hline
\end{tabular}

The responses of the $1^{\text {st }}$ and final years MBBS students pertaining to the leadership domains and their associated competencies varied as shown in Figures-1 \& 2. In terms of the leadership domain 'demonstrating personal qualities', a substantial number of students $(n=77)$ from 1st year chose to manage themselves and continuing professional development as essential components of this domain (Figure- 1 ). In this $50 \%$ of the first-year students were of the view that ensuring patient safety was an important component of the leadership domain of improving services whereas only $11 \%$ responded as critically evaluating as a component of this domain.

On the contrary, $77 \%$ of final-year students responded ensuring patient safety is a component of the leadership domain improving services. Out of $58 \%$ of the final year, students were of the view that managing people is an important leadership domain of 'mana-ging services' as depicted in Figure-2.

\section{Themes Emerging from the Focus Group Discussions}

In total 3 focus group discussions were conducted among the students comprising of groups of 12

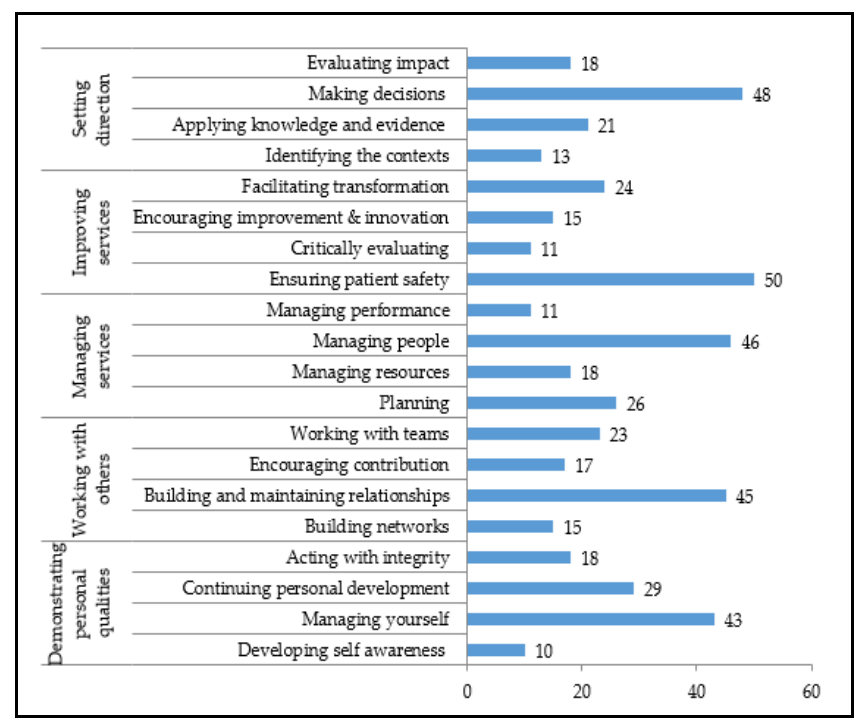

Figure-1: Five Leadership Domain and its associated competencies: Percentage responses by 1st year medical students $(\mathbf{n}=107)$.

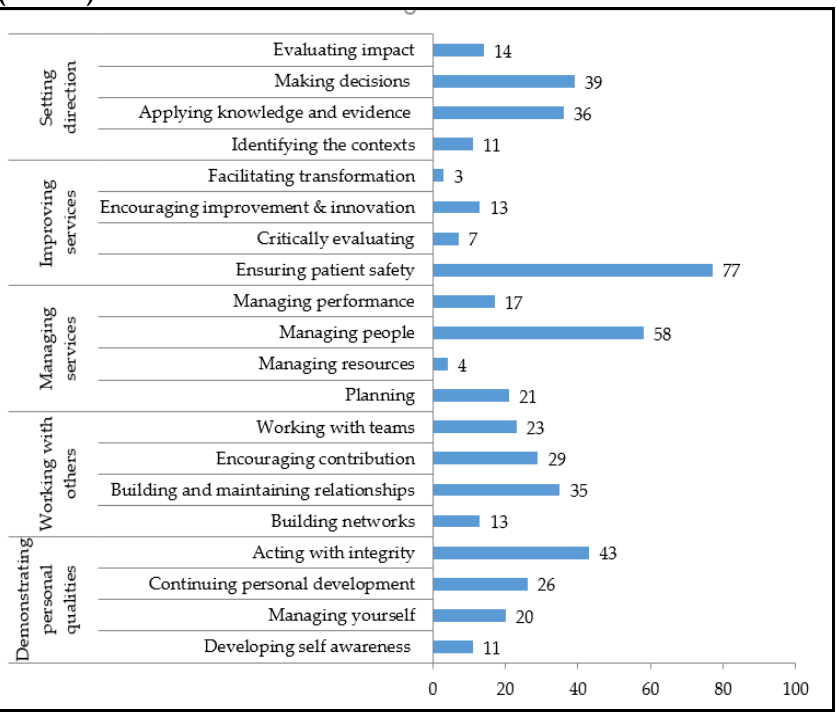

Figure-2: Five Leadership domain and its associated competencies: percentage responses by final year medical students $(n=100)$.

students. The groups had an equal proportion of male and female students representing both first-year and final year students. The focus group discussions were guided by a focus group guide comprising of important components, mainly encompassing questions pertaining to views about the competencies included in leadership domains, the role of faculty members and clinical teachers in implementing leadership domains within curriculum and handson practice, the facilitators and challenges in meeting the desired targets, effective implementation and internalization of concepts and the most prudent way forward. The themes that emerged after analysis included; increasing awareness, 
role models depicting change, cultural challenges, and supplementing existing practices. The inter-relation of the various themes is depicted in Figure-3.

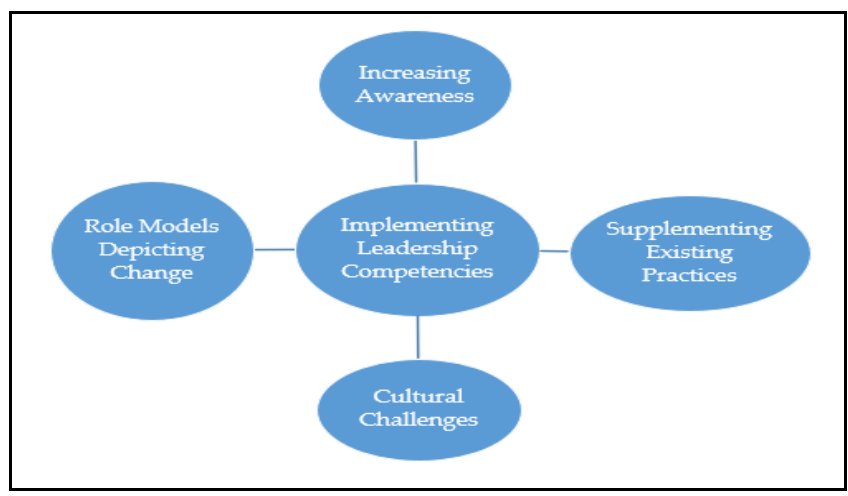

Figure-3: Important themes associated with implementing Leadership competencies among the undergraduate medical students.

\section{Increasing Awareness}

Students discussed and highlighted the significance of increasing awareness pertaining to the leadership competencies among the undergraduate medical students and considering it to be included as an essential part of the taught curriculum. Students were of the view that mostly unaware of leadership domain and the associated competencies within them. They never considered the fact that graduates are supposed to be equipped with the essential skills and competencies which could aid them in becoming effective leaders within managing healthcare systems and practising clinical skills to ensure maximum benefit to the patients. The students discussed the need to explicitly include leadership competencies within the curriculum starting by firstly increasing awareness among newly enrolled students in the medical college.

A first-year female medical said while discussing how to increase awareness regarding building leadership competencies among undergraduate students: 'It is a surprise for me to at least realise that developing leadership skills is important for a doctor. I was of the view that only business studies or management students were taught such skills. I think it is very important to highlight its importance right from the start. I mean in the very beginning when a medical student is enrolled and starts his journey in medicine'.

\section{Role Models Depicting Change}

It was emphasized that faculty members and clinical physicians can play a significant role in displaying the various attributes associated with leadership through their day to day engagements with the students and patients. The students were of the view that the trainer can act as a role model which can create a great impact in ensuring that the competencies are well registered in the minds of the undergraduate trainees.

\section{A Male Final Year Mbbs Student Said}

'Students learn the most by silently observing their instructors during lectures, bedside teaching and other interactions related to the course. If a student doesn't observe professional behaviour and all the competencies associated with leadership among his instructors and teaching faculty it is very unlikely that he/she would ever be able to deeply understand and appreciate its significance in becoming a competent doctor.

\section{Cultural Challenges}

It was highlighted during the focus group discussions that the cultural background of the students and the faculty can pose a challenge in understanding the various competencies associated with leadership and applying them in clinical settings. It was discussed that the background and previous exposures can play an important role in determining desired outcomes.

\section{A Final Year Female Student Commented}

'It will depend a lot on the upbringing and experiences of an individual. Not everyone is born a leader. Yes, the leadership competencies are defined but I think there is more to it. Certain individuals are just more caring by nature and this may be associated strongly with their cultural background. Some competencies may be more refined in some individuals as compared to others. In addition, some more time and hard work are required to adopt the competencies within broadly defined leadership domains'.

\section{Supplementing Existing Practices}

The students acknowledged that some form of leadership competencies were practised by the faculty and instructors. In addition, they felt it would be prudent to build on existing practices to streamline and clearly define all the domains of leadership and the competencies within each domain.

\section{DISCUSSION}

The main findings of the study included varied responses pertaining to the leadership domains and their associated competencies with a significant percentage of final-year students reporting patient safety as an important indicator. First-year undergraduates had similar percentage responses to final year students pertaining to managing people and relationships among peers and teaching staff competencies within 
leadership domains. The qualitative findings mainly suggested cultural challenges, building on existing practices to promote leadership competencies and the teaching and clinical staff acting as role models to instigate essential leadership competencies as important aspects and strategies to enhance leadership competencies among medical undergraduate students. The various strategies that have been practised ad implemented for the capacity building of physicians and undergraduate medical students in context to leadership and management include; developing leadership through service learning 11 , workshops ${ }^{8}$, online courses in form of designed modules ${ }^{8}$, short courses ${ }^{12}$, integration of medical degree with a business management $t^{4}$, collaboration of business schools with medical schools 13, modelling leadership curriculum ${ }^{5}$, elective programmes for a better understanding of health management and leadership roles ${ }^{14}$, fellowships and longitudinal programs for the development of leadership competencies ${ }^{8}$.

This study highlights expected and desired competencies at the entry and exit level of the medical undergraduate course, which fills the gap in the literature as studies ${ }^{11}$, have been conducted to assess student's perceptions about leadership and management but no significant research study has been conducted to assess the expected or desired competency level at entry-level and after graduation specifically. The findings of the research highlight the socio-cultural aspects as expressed in the qualitative findings how they can affect the attainment of the various competencies and also pose a challenge. These findings are substantiated by the fact that in low middle-income country settings which is resource-limited and have weakened health infrastructure substantial research is essential to define the core leadership competencies ${ }^{17-20}$, and assess the competency level of the undergraduate medical students to propose an innovative future skillbased leadership competency program to be included comprehensively in the medical curriculum which should ideally be tailored in accordance to the service delivery needs of the specific communities.

The study also highlights the main leadership domains and their significance among the medical undergraduates. These domains and competencies can be prioritized according to the training year of the student. Literature suggests that although the short course and guidelines are mentioned in MBBS curriculum, postgraduate, community outreach programs or nursing training programmes of some institutes such as Agha Khan University ${ }^{20}$, and Shifa International Medical College ${ }^{24}$, a substantial gap remains in a comprehensive evidence-based program encompassing Leadership. In Pakistan, there is a lack of an evidencebased curriculum designed to develop leadership competencies among medical undergraduates aimed at community-based service delivery. The findings add to bridge these gaps in the knowledge base.

The diverse and multifaceted nature of the leadership competencies described by the findings of the study is also similar to findings of the various studies412 , which suggest that there is a need to be streamlining leadership competencies according to the training level and context of the expected desired outcomes. There is a gap in the contextualization and defining of the core leadership competencies particularly among undergraduate medical students both at the entry-level and during graduation. The perceptions among the different genders of medical undergraduates also require due consideration while understanding the basic leadership roles ${ }^{16}$. This fact was also highlighted in our findings. This is further reinforced by the fact that although attempts have been made to incorporate leadership and management in the undergraduate medical curriculum it has been reported to be insufficient and not catering to the felt needs of the undergraduate students ${ }^{5,11}$. Furthermore, a systematic review of 26 studies 11 pertaining to perceptions of medical undergraduates and faculty to core leadership competencies highlighted that little emphasis is given to leadership and management in the medical curriculum. This is strongly reflected by the responses provided by the students in focus group discussions, a lack of structure and clear emphasis on the significance of these competencies during lectures and clinical teaching.

Leadership competencies among undergraduate medical students are essential for service delivery particularly in resource-limited weakened health infrastructures for increasing quality of care and efficient team management. The leadership competencies are not defined in the context of desired outcomes once the medical students graduate. The students need to learn and adapt their leadership competencies in accordance with the needs of the services they would be providing after their graduation in their defined socio-cultural settings considering the existing challenges by applying region-specific and innovative interventions.

\section{ACKNOWLEDGEMENT}

The authors would like to thank the medical students of Fatima Memorial Hospital Medical and Dental College for 
participating in the study. The corresponding author would also like to thank the teaching staff of the College of Physicians and Surgeons Pakistan in the Health Professionals Education Membership program for providing valuable input during the proposal development and approval process.

\section{CONCLUSION}

The study highlights the validated essential domains of leadership and their associated competencies should be streamlined and prioritised in accordance with the level of training of the undergraduate medical students. Their input is important as they are direct beneficiaries and the highlighted challenges should be carefully considered to achieve desired outcomes by the active involvement of the teaching staff. A multicentre study is suggested to gather data at the national level which could inform further development and active implementation of leadership domains within the undergraduate MBBS curriculum.

\section{Conflict of Interest: None.}

\section{Author's Contribution}

SMA: Data collection, data analysis, drafting of protocol, results and final manuscript. AA: Drafting of protocol, drafting of the final manuscript and final review of the paper.

\section{REFERENCES}

1. Creswell J. Research Design: Qualitative, Quantitative and Mixed Methods Approaches. 2nd ed. Thousand Oaks CA: Sage, 2003, [Internet] Available at Internet: http://www.drbrambedkarcollege.ac.in/sites/default/files/Research-Design_QualitativeQuantitative-and-Mixed-Methods-Approaches.pdf

2. Parker M. Misconceiving medical leadership. Perspect Biol Med. Summer; 2013; 56(3): 387-406.

3. Bleich MR. Leadership needs assessment. J Contin Educ Nurs 2015; 46(1): 10-15.

4. Sherrill WW. Dual-degree MD-MBA. Students: A Look at the Future of Medical Leadership. Acad Med 2000; 75(10): S37-S39.

5. Crites GE, Ebert JR, Schuster RJ. Beyond the dual degree: Development of a five-year program in leadership for medical undergraduates. Acad Med 2008; 83(1): 52-58.

6. Connell MT, Pascoe JM. Undergraduate medical education for the $21^{\text {st }}$ century: leadership and teamwork. Family MedicineKansas City 2004; 36(Supply-I): S51-S56.

7. Goldstein AO, Calleson D, Bearman R, Steiner BD, Frasier PY. Teaching advanced leadership skills in community service (ALSCS) to medical students. Acad Med 2009; 84(6): 754-764.

8. Steinert $Y$, Naismith L. Faculty development initiatives designed to promote leadership in medical education. A BEME systematic review: BEME Guide No. 19. Med Teach 2012; 34(6): 483-503.
9. Clyne B, Rapoza B, George P. Leadership in Undergraduate Medical Education: Training Future Physician Leaders. R I Med J 2013; 98(9): 36-40.

10. Abbas MR, Quince TA, Wood DF, Benson JA. Attitudes of medical students to medical leadership and management: a systematic review to inform curriculum development. BMC Med Edu 2011; 11(1): 93-98.

11. Long JA, Lee RS, Federico S, Battaglia C. Developing leadership and advocacy skills in medical students through servicelearning. J Public Health Manage Prac 2011; 17(4): 369-372.

12. Smith KL, Petersen DJ, Soriano R, Friedman E, Bensinger LD. Training tomorrow's teachers today: a national medical student teaching and leadership retreat. Med Teach 2007; 29(4): 328-334.

13. Larson DB, Chandler M, Forman HP. MD/MBA programs in the United States: evidence of a change in health care leadership. Acad Med 2003; 78(3): 335-341.

14. Agarwal A, Anderson J, Sarfaty S, Rimer E, Hirsch AE. The Value of an Elective in Business and Leadership for Medical Students. J Med Pract Man 2015; 30(4): 276-280.

15. Ariff S, Soofi SB, Sadiq K, Feroze AB, Khan S, Jafarey SN, et al. Evaluation of health workforce competence in maternal and neonatal issues in the public health sector of Pakistan: an assessment of their training needs. BMC Health Ser Res 2010; 10(1): 319-323.

16. Silva A. What is Leadership. J Bus Stu Quart 2016; 8(1): 1-8.

17. Cothran DJ. Creating curriculum to enhance student learning. Routledge Handbook of Physical Education Pedagogies 2016: 39.

18. Bolden R. Is the NHS Leadership Qualities framework missing the wood for the trees. Innovations in Health Care: A Reality Check. Basingstoke: Palgrave Macmillan. 2006.

19. Clark J, Armit K. Leadership competency for doctors: a framework. Lead Health Ser 2010; 23(2): 115-129.

20. Frank JR, Mungroo R, Ahmad Y, Wang M, De Rossi S, Horsley T. Toward a definition of competency-based education in medicine: a systematic review of published definitions. Med Teacher 2010; 32(8): 631-637.

21. NHS Leadership academy: Clinical leadership competency framework; 2011 [Internet] Available from: https://www.l eadershipacademy.nhs.uk/wpcontent/uploads/2012/11/ NHSLeadership-Leadership-Framework-Clinical-LeadershipCompetency-Framework-CLCF.pdf [cited 2017 Dec 24].

22. Gale NK, Heath G, Cameron E, Rashid S. Using the framework method for the analysis of qualitative data in multi-disciplinary health research. BMC Med Res Methodol 2013; 13(1): 117.

23. Tong A, Sainsbury P, Craig J. Consolidated criteria for reporting qualitative research (COREQ): a 32-item checklist for interviews and focus groups. Int J Qual Health Car 2007; 19(6): 349-357.

24. Bryant JH, Marsh DR, Khan KS, D'Souza R, Husein K, Aslam A, et al. A developing country's university oriented toward strengthening health systems: challenges and results. Am J Public Health 1993; 83(11): 1537-1543. 